DOI: http://dx.doi.org/10.12957/demetra.2014.10383

\title{
Necessidades alimentares especiais em ambiente escolar: um ensaio sobre a interface entre ciências da Nutrição e Bioética
}

\section{Special dietary requirements in the school environment: an essay on the interface between Nutrition and Bioethics}

\author{
Cilene da Silva Gomes Ribeiro' \\ Maria Teresa Gomes de Oliveira Ribas' \\ Carla Corradi-Perini² \\ Flavia Auler' \\ ' Curso de Nutrição, Escola de Saúde e \\ Biociências. Pontifícia Universidade Católica do \\ Paraná. Curitiba-PR, Brasil. \\ 2 Programa de Pós-Graduação em Bioética, \\ Escola de Saúde e Biociências, \\ Pontifícia Universidade Católica do Paraná. \\ Curitiba-PR, Brasil. \\ Correspondência /Correspondence \\ Cilene da Silva Gomes Ribeiro \\ E-mail: cilenex@hotmail.com
}

\section{Resumo}

Este ensaio busca levantar elementos de discussão a respeito de estratégias de políticas públicas para o atendimento das necessidades alimentares especiais no ambiente escolar, à luz da interdisciplinaridade das ciências da Nutrição e da Bioética, na perspectiva da segurança alimentar e nutricional e do direito humano à alimentação adequada. A reflexão tem a expectativa de motivar o desenvolvimento de linhas e redes de pesquisa interinstitucionais, com a finalidade de contribuir para a produção e implementação de indicadores de segurança alimentar e nutricional no âmbito da gestão de políticas públicas, no contexto das necessidades alimentares especiais.

Palavras-chaves: Necessidades Alimentares Especiais. Alimentação Escolar. Segurança Alimentar e Nutricional. Nutrição. Bioética. Direito Humano à Alimentação Adequada. 


\section{Abstract}

This essay aims to identify elements of discussion of strategies for public policies to meet the special dietary needs in the school environment, in light of the interdisciplinarity of Nutrition Science and Bioethics, in view of the food and nutritional security and the human right to adequate food. This reflection expects to motivate development of lines and inter-institutional research networks, contributing to the production and implementation of nutritional and food security indicators for the management of public policies in the context of special dietary needs.

Key words: Special Dietary Needs. School Food. Food and Nutritional Security. Nutrition. Bioethics. Human Right to Adequate Food.

\section{Introdução}

O presente ensaio busca discutir estratégias de políticas públicas para o atendimento das necessidades alimentares especiais no ambiente escolar, à luz da interdisciplinaridade da Alimentação e Nutrição e da Bioética, na perspectiva da segurança alimentar e nutricional e do direito humano à alimentação adequada (DHAA).

A alimentação, por atender a uma das necessidades básicas do homem, a sobrevivência, sempre foi objeto de preocupação individual e coletiva. Deste modo, no Brasil, a realização do direito à alimentação - a partir da promulgação na Emenda Constitucional 64, que inclui a alimentação entre os direitos sociais, fixados no artigo $6^{\circ}$ da Constituição Federal no dia 05 de fevereiro de 2010, ${ }^{1}$ foi referenciada na perspectiva de estratégias que visam à Segurança Alimentar e Nutricional (SAN). Ou seja, ratifica as dimensões da concepção brasileira de SAN, que dizem respeito à "garantia do direito de todos ao acesso regular e permanente a alimentos de qualidade, em quantidade suficiente, sem comprometer o acesso a outras necessidades essenciais, tendo como base práticas alimentares promotoras da saúde, que respeitem a diversidade cultural e que sejam ambiental, econômica e socialmente sustentáveis". ${ }^{2}$

A SAN é uma temática de política pública que está em processo de consolidação no Brasil, trazendo bases para a inclusão da questão do DHAA na agenda política e na gestão pública mediante a realização sistemática de conferências nacionais e a estruturação dos marcos legais da política nacional que sustentam a configuração do Sistema e do Plano Nacional de Segurança Alimentar e Nutricional (SISAN) ${ }^{3-7}$ As ações de SAN têm como um dos princípios a articulação entre o governo 
e a sociedade para a formulação de políticas e na definição de orientações para que o país garanta o direito humano à alimentação - a fim de fazer cumprir o dever do poder público em respeitar, proteger, promover, prover, informar, monitorar, fiscalizar e avaliar a realização do direito humano à alimentação adequada, bem como garantir os mecanismos para sua exigibilidade. ${ }^{3}$

Através dos princípios de abrangência, intersetorialidade, equidade, participação social e articulação entre medidas de caráter emergencial e estrutural, se conforma a Política Nacional de Segurança Alimentar e Nutricional. ${ }^{8}$

As diretrizes dessa política estão organizadas no sentido de atender a oito dimensões, dentre as quais se destacam as seguintes, pela forte correlação com as intenções da proposta de pesquisa apresentada: promoção do acesso universal à alimentação adequada e saudável, com prioridade para as famílias e pessoas em situação de insegurança alimentar e nutricional; promoção do abastecimento e estruturação de sistemas sustentáveis e descentralizados, de base agroecológica, de produção, extração, processamento e distribuição de alimentos; instituição de processos permanentes de educação alimentar e nutricional, pesquisa e formação nas áreas de segurança alimentar e nutricional e do direito humano à alimentação adequada; fortalecimento das ações de alimentação e nutrição em todos os níveis da atenção à saúde, de modo articulado às demais ações de segurança alimentar e nutricional; e monitoramento da realização do direito humano à alimentação adequada. ${ }^{3}$

Dentre as estratégias basilares para a ação do governo nesse contexto, pode-se mencionar a organização da atenção nutricional em todos os níveis do Sistema Único de Saúde (SUS) e o Programa Nacional de Alimentação Escolar (PNAE).

Quanto ao marco legal referente à abordagem da alimentação no escopo do SUS, a Lei $n^{\circ} 8.080$, de 19/09/1990, ${ }^{9}$ evidencia em seu conteúdo o componente alimentar no processo de determinação da saúde-doença e, portanto, como estratégia transversal às ações formuladas e executadas sob a responsabilidade assistencial do Estado, que devem ser avaliadas continuamente no âmbito do sistema público de saúde.

Assim, mediante o conteúdo do instrumento jurídico acima referido, em associação à publicação da primeira versão da Política Nacional de Alimentação e Nutrição (PNAN),${ }^{10}$ considera-se possível evidenciar um espaço inicial de identidade da relação entre alimentação e saúde na operacionalidade do SUS. Destaca-se que esse processo demarca uma perspectiva em direção à realização das ações de alimentação e nutrição para além das atividades curativas e assistenciais, mas avançando para a exigência da intersetorialidade no enfrentamento dos determinantes da saúde e nutrição na população. Fica estabelecida a concretude dessas ações na efetivação da vigilância alimentar e nutricional e nas estratégias de orientação alimentar nos planos individual e coletivo. A 
partir desse espaço legitimado, podem ser delineados os campos de assistência, educação e gestão para as ações de nutrição em saúde. ${ }^{11}$

No contexto do SUS, a Política Nacional de Alimentação e Nutrição (PNAN) tem como uma das prioridades a promoção da alimentação adequada, que envolve a prática alimentar apropriada aos aspectos biológicos e socioculturais dos indivíduos, bem como o uso sustentável do meio ambiente, devendo respeitar as necessidades de cada fase do curso da vida. Ressalta-se que, ainda nessa perspectiva, a PNAN assume como demandas para a atenção nutricional no SUS o cuidado aos indivíduos com necessidades alimentares especiais. ${ }^{12}$

Tem-se reconhecido que, após uma década de sua publicação, a PNAN enfrenta desafios complexos quanto às exigências na proposição e efetivação de soluções para o atendimento da trama de dimensões que envolvem os impactos sobre a alimentação e nutrição no campo da Saúde Coletiva. Nesse sentido, cada vez mais se impõe sua articulação com a Política Nacional de Segurança Alimentar e Nutricional. ${ }^{13}$

Dentre as demandas complexas em nutrição e saúde, é possível identificar as situações do curso da vida onde se evidenciam alterações metabólicas e fisiológicas que requerem alterações nas necessidades alimentares dos indivíduos. Desse modo, a PNAN prevê a assistência a essas situações consideradas como "necessidades alimentares especiais":

[...] necessidades alimentares, sejam restritivas ou suplementares, de indivíduos portadores de alteração metabólica ou fisiológica que cause mudanças, temporárias ou permanentes, relacionadas à utilização biológica de nutrientes ou a via de consumo alimentar (enteral ou parenteral). Exemplos: erros inatos do metabolismo, doença celíaca, HIV/Aids, intolerâncias alimentares, alergias alimentares, transtornos alimentares, prematuridade, nefropatias, etc.

Necessidades alimentares especiais nas ações da política de alimentação escolar: caminhos para visibilidade, subjetividade e inclusão

Dentro do cenário da alimentação escolar, de acordo com um dos princípios que norteiam o PNAE, evidencia-se a equidade como fundamento para a promoção do "tratamento igual para os alunos sadios e o tratamento diferenciado para os alunos portadores de necessidades especiais, a exemplo dos celíacos e dos diabéticos, que devem receber uma alimentação adequada à sua condição, por intermédio de cardápio elaborado por nutricionista habilitado". ${ }^{14}$ Considerase essa decisão como um processo fundante da visibilidade da questão das NAE no contexto das políticas públicas. 
Outro marco legal importante, enquanto proposição de avanços intersetoriais em direção à qualidade da alimentação e seus impactos na saúde e nutrição escolar, diz respeito à Portaria Interministerial n⿳0 1.010, de 08 de maio de 2006, que institui as diretrizes para a Promoção da Alimentação Saudável nas Escolas de educação infantil, fundamental e nível médio das redes públicas e privadas, em âmbito nacional. ${ }^{15}$

Mas pode-se considerar que a principal conquista recente na ótica da SAN no cenário da alimentação escolar diz respeito à aprovação de marco legal referente à articulação do PNAE com a agricultura familiar no fornecimento dos produtos para a assistência alimentar, o que inclui a priorização de alimentos produzidos em modelos de sistemas alimentares agroecológicos. ${ }^{16}$ A Lei n ${ }^{\circ}$ 11.947/2009 dispõe sobre a obrigatoriedade de estados e municípios utilizarem no mínimo 30\% dos recursos repassados pelo Governo Federal na compra de gêneros advindos da agricultura familiar. No Paraná, a contratação e o fornecimento dos gêneros são efetuados a partir de organizações representativas dos agricultores familiares, tais como cooperativas ou associações, obrigatoriamente portadoras de DAP Jurídica (Declaração de Aptidão ao Pronaf). ${ }^{17}$

Com relação à dimensão das subjetividades presentes na experiência vivida dos sujeitos com necessidades alimentares especiais, tem-se que instituições ligadas à sua proteção e assistência têm-se posicionado em relação ao fato de que a vivência de restrições alimentares sem o direito de acesso a alimentos adequados, a partir de políticas públicas, configura fator de exclusão social. Nessa concepção, tal fato se caracteriza como situação de insegurança alimentar e nutricional, agravada pelas restrições sociais que se impõem às pessoas com deficiência orgânica.

Desse modo, tem sido possível diagnosticar que, a partir de políticas públicas voltadas à segurança alimentar e nutricional, foi aberto um campo para a evidenciação da problemática do direito humano à alimentação adequada para pessoas com necessidades alimentares especiais. Conforme descrito, no âmbito das políticas setoriais com interface na assistência alimentar e foco na SAN, o Programa de Alimentação Escolar tem apresentado proposições que podem representar avanços em direção à atenção a sujeitos com necessidades alimentares especiais.

Com os recentes aprimoramentos de marcos legais de políticas públicas e de resoluções específicas que evidenciam competências do nutricionista no âmbito de intervenções de promoção da saúde no ambiente escolar, tem havido a preocupação em dar institucionalidade a ações de articulação entre esses profissionais e educadores na realização de ações conjuntas de educação alimentar e nutricional para a comunidade escolar. Dentro desse foco, documentos orientadores têm sido produzidos na ótica de desenvolvimento e operacionalização das atividades inerentes ao PNAE, relacionadas ao fornecimento de alimentação escolar para atendimento das necessidades de sujeitos com diabetes, hipertensão, doença celíaca, fenilcetonúria e intolerância à lactose. ${ }^{12}$ 
No cenário nacional, têm sido identificadas experiências consoantes à perspectiva de promoção da SAN em ambiente escolar e que dizem respeito à implantação do Sistema de Vigilância Alimentar e Nutricional Escolar (SISVAN Escolar), como instrumento de monitoramento do perfil nutricional e planejamento de ações de prevenção e controle da obesidade e de doenças crônicas não transmissíveis. Outro passo importante tem sido a identificação de escolares com necessidades alimentares especiais, no cadastramento de redes públicas de ensino. ${ }^{18} \mathrm{O}$ mapeamento desses escolares é o primeiro passo para a implementação de estratégias que visem atender a suas necessidades.

A presença de crianças com necessidades alimentares especiais também tem sido foco de reflexões importantes no campo da inclusão educacional. Ou seja, estudos tratam de redimensionar a discussão no interior das redes de ensino a respeito das dimensões da educação alimentar escolar, na perspectiva de práticas inclusivas.

Esse panorama evidencia a necessidade de implementar estratégias de fortalecimento intersetorial de educação e saúde, na direção de promover a segurança alimentar e nutricional para atendimento das necessidades alimentares especiais, incluindo todos os atores do cenário escolar. Ao mesmo tempo, sinalizam especialmente, para além da esfera médica, a relevância da identificação e análise das diversas dimensões do desfecho nutricional daqueles com necessidades alimentares especiais, mais vulneráveis às situações de insegurança alimentar e nutricional. ${ }^{19}$

Dentre os desafios que podem ser apontados para a produção de conhecimento e que representam lacunas presentes no âmbito das políticas públicas, podem ser citados a necessidade de compreensão aprofundada sobre a complexidade da experiência vivida no cotidiano das pessoas com necessidades alimentares especiais, ampliando sua dimensão para além dos aspectos biomédicos; a avaliação das estratégias de assistência alimentar vigentes, incluído o seu impacto nutricional; e o fortalecimento de ações para monitoramento das violações do DHAA dos sujeitos em tela.

\section{Atendimento às necessidades alimentares especiais no ambiente escolar: uma questão bioética}

O contexto e o momento histórico em que vivemos fazem com que a bioética se debruce sobre as transformações políticas, econômicas, sociais, culturais, ambientais, epidemiológicas e demográficas, para uma aproximação com os problemas da coletividade, principalmente em países com grandes níveis de desigualdade social, como o Brasil. ${ }^{20}$ Neste sentido, há necessidade de ampliar o olhar da bioética para questões concretas existentes em nosso país, incluindo as relacionadas à qualidade da vida humana, como a alimentação adequada. Uma bioética que se oriente pelo respeito e incentivo à liberdade individual de tomada de decisão, adicionada dos princípios da 
solidariedade, da justiça, da equidade e da responsabilidade, reforçando a necessidade de proteção dos mais desfavorecidos, vulneráveis, vulnerados ou frágeis. ${ }^{20}$

É imperativo que a bioética e seus referenciais sejam incorporados nos processos de definição das políticas públicas, contribuindo na construção das sociedades que garantam os direitos humanos, pois se fundamenta numa visão macro, ampliada e seguramente comprometida com a dimensão social. ${ }^{21} \mathrm{~A}$ interdisciplinaridade, condição fundamental para a Bioética, a torna um campo peculiarmente interessante de se debater as questões relacionadas aos direitos humanos, pois se não houver o enfrentamento da realidade na qual os mesmos serão aplicados, a simples adoção de princípios universais é inadequada. ${ }^{22}$

No âmbito das necessidades alimentares especiais, especificamente, afirma-se a insuficiência de considerar exclusivamente a integridade e dignidade da pessoa para a implementação de estratégias que visem assegurar o DHAA a essa população. É necessário reconhecer que este e outros direitos apenas poderão ser plenamente alcançados numa sociedade com equidade na distribuição dos bens e nos acessos a condições de vida digna. ${ }^{23} \mathrm{~A}$ inserção da bioética no campo do DHAA fica evidente no artigo 14 da Declaração Universal sobre Bioética e Direitos Humanos, "Responsabilidade Social e Saúde", que trata da questão do acesso às condições essenciais para a sobrevivência física e social das populações humanas, no item ii, "Acesso à nutrição adequada e água de boa qualidade". ${ }^{24}$

Assim, o DHAA não pode ser reduzido a uma mera concepção normativa. Os direitos humanos são construídos sobre uma base dinâmica de respeito, inclusão e reconhecimento. ${ }^{25,26}$ Para a incorporação do conceito de DHAA nas estratégias de desenvolvimento social e de SAN, são necessárias mudanças nos processos, nas formas de fazer as coisas e, fundamentalmente, na cultura institucional do Estado, de seus servidores e da população em geral. ${ }^{27,28}$ Destaca-se ainda a urgência de se operacionalizar avaliações de políticas públicas de SAN, como por exemplo o PNAE, para que as mesmas estejam integradas aos princípios que regem a abordagem baseada nos direitos humanos e que reconheçam o indivíduo como sujeito de direitos. ${ }^{29}$

Siqueira et al.,,$^{30} \mathrm{em}$ sua discussão sobre a incorporação da perspectiva do DHAA no desenho institucional do PNAE, afirmam que esta política de SAN começou a substituir a visão de uma política de caráter assistencialista e de transferência de recursos, para uma concepção do benefício como um direito que deve ter controle social. Entretanto, persistem ainda inúmeros obstáculos, anteriormente descritos, que dificultam sua concretização enquanto medida de promoção do DHAA. 
Siqueira et al. ${ }^{30}$ sugerem que um dos fatores que proporcionam a persistência desses obstáculos é o fato de que o PNAE é um programa de larga abrangência onde, inevitavelmente, as mudanças transcorrem com ritmo e impacto distinto e condicionado a interesses sociais, políticos e econômicos dos estados e municípios. Particularmente, no Estado do Paraná, tem-se observado avanços em relação à promoção do DHAA no campo da alimentação escolar. Essa constatação tem sustento, mais especificamente, a partir da observância de iniciativas na gestão pública do setor de educação que incluem o cadastramento de alunos com necessidades alimentares especiais e a articulação com o Programa de Aquisição de Alimentos, propiciando, respectivamente, oferta de uma alimentação diferenciada e mais adequada para esta população. Além da avaliação da composição corporal, através do peso e altura dos escolares, em 2011 foram incorporados ao monitoramento, a verificação da incidência de alunos com diabetes, intolerância à lactose e doença celíaca, situações que envolvem necessidades alimentares especiais.

Essas iniciativas, ainda que insuficientes para assegurar plenamente o DHAA dos sujeitos em tela, representam sinais da incorporação de alguns referenciais da Bioética, tais como a vulnerabilidade, ${ }^{31}$ a equidade ${ }^{32} \mathrm{e}$ a justiça ${ }^{33}$ nas ações do Estado no que tange à alimentação escolar. Especificamente, pode-se afirmar que o reconhecimento da vulnerabilidade determinada por uma disfunção orgânica apresentada pelos escolares com diabetes, intolerância à lactose e doença celíaca, traduz-se em ações equitativas. Ou seja, a "equidade cuida de igualdade na medida em que trata como igual o que é igual, mas, quando necessário, tratam de modo desigual (porém, o adequado) o que é desigual", ${ }^{31}$ o que às vezes é necessário para se atingir a igualdade. Portanto, em se tratando dos escolares com necessidades alimentares especiais, podemos afirmar que todos têm igual direito à alimentação, mas devemos atender às diferentes necessidades. Tratando de forma desigual (porém, adequada) os escolares com necessidades alimentares especiais (desiguais), praticase a justiça, entendida como uma virtude primária das instituições sociais, fruto da cooperação humana que deve pretender a realização de benefícios mútuos. ${ }^{34}$

\section{Considerações finais}

Apesar do aumento de interesse pela investigação científica sobre o tema das necessidades alimentares especiais, há necessidade de haver investimentos ampliados para projetos de pesquisa no meio acadêmico, especialmente no que tange a situar a temática das necessidades alimentares especiais na perspectiva do DHAA em especial, a partir de sua inclusão como campo assistencial de responsabilidade da Política Nacional de Alimentação escolar. Reconhece-se, assim, a relevância do fomento dessa linha de pesquisa em seu potencial de qualificação de estratégias em direção ao alcance da SAN no cenário das necessidades alimentares especiais. 
A partir deste ensaio, é possível levantar, no campo da produção de conhecimento científico em Nutrição, importantes dimensões de análise da questão das NAE em ambiente escolar. Estas envolvem a qualidade nos processos de produção e oferta alimentar, aspectos referentes ao desfecho nutricional dos sujeitos com necessidades alimentares especiais, subjetividades relativas à alimentação e nutrição no contexto da vivência no ambiente escolar das patologias que requerem restrições alimentares e institucionalidade do direito humano à alimentação adequada.

Ainda no campo da Bioética, propõe-se a adoção da perspectiva inclusiva, com uma visão do escolar associado aos seus vínculos comunitários, em um contexto social, econômico e cultural. Sendo assim, a denegação ou violação de seus direitos, mais particularmente, o direito humano à alimentação adequada, pode afetar esses escolares com necessidades alimentares especiais, em todas as esferas de suas vidas.

\section{Referências}

1. Brasil. Emenda Constitucional n ${ }^{\circ} 64$ de 4 de fevereiro de 2010. Altera o artigo $6^{\circ}$ da Constituição Federal para introduzir a alimentação como direito social. Diário Oficial da União 5 fev. 2010; 1.

2. Brasil. Lei no 11346, de 15 de setembro de 2006. Cria o Sistema Nacional de Segurança Alimentar e Nutricional - SISAN com vistas em assegurar o direito humano à alimentação adequada e dá outras providências. Diário Oficial da União 18 set. 2006.

3. Brasil. Decreto $n^{\circ} 7.272$ de 25 de agosto de 2010. Regulamenta a Lei $n^{\circ}$ 11.346, de 15 de setembro de 2006, que cria o Sistema Nacional de Segurança Alimentar e Nutricional - SISAN com vistas a assegurar o direito humano à alimentação adequada, institui a Política Nacional de Segurança Alimentar e Nutricional - PNSAN, estabelece os parâmetros para a elaboração do Plano Nacional de Segurança Alimentar e Nutricional, e dá outras providências.. Diário Oficial da União 26 ago. 2010; 1:6.

4. Brasil. Conselho Nacional de Segurança Alimentar e Nutricional. Princípios e diretrizes de uma poítica de segurança alimentar e nutricional. Textos de referência da II Conferência nacional de Segurança Alimentar e Nutricional. Brasília: Consea; 2004.

5. Brasil. Conselho Nacional de Segurança Alimentar e Nutricional. A construção da política nacional de segurança alimentar e nutricional. II Conferência Nacional de Segurança Alimentar e Nutricional. Relatório Final - $2^{a}$ versão. Olinda: Consea; 2004.

6. Brasil. Câmara Interministerial de Segurança Alimentar e Nutricional. Plano nacional de segurança alimentar e nutricional - 2012/2015. Brasília: Caisan; 2011

7. Brasil. Conselho Nacional de Segurança Alimentar e Nutricional. Alimentação adequada e saudável: direito de todos. 4a Conferência Nacional de Segurança Alimentar e Nutricional. Salvador: Consea; 2011. Documento Base, 2011.

8. Brasil. Ministério da Saúde. Protocolos do sistema de vigilância alimentar e nutricional - SISVAN na assistência à saúde. Brasília: Ministério da Saúde; 2008. 61 p. 
9. Brasil. Lei no 8080, de 19 de setembro de 1990. Dispõe sobre as condições para promoção, proteção e recuperação da saúde, a organização e o funcionamento dos serviços correspondentes e dá outras providências. Diário Oficial da União 20 set. 1990; 1:18055.

10. Brasil. Ministério da Saúde. Política nacional de alimentação e nutrição. Brasília: Ministério da Saúde; 2000.

11. Lang RMF, Ribas MTGO. O nutricionista e as ações de nutrição em saúde. In: Taddei, JAAC, Lang RMF, Longo-Silva G, Toloni MHA. Nutrição em saúde pública. Rio de Janeiro: Rubio; 2011. p. 437-453.

12. Brasil. Ministério da Saúde. Política nacional de alimentação e nutrição. Brasília: Ministério da Saúde; 2012. 84 p.

13. Recine E, Vasconcellos, AB. Políticas nacionais e o campo da alimentação e nutrição em saúde coletiva: cenário atual. Ciênc. Saúde Coletiva 2011; 16(1):73-79.

14. Brasil. Lei de segurança alimentar e nutricional [Internet]. [acesso em 5 ago. 2013]. Disponível em: http://www.planalto.gov.br/ccivil_03/_ato2004-2006/2006/lei/111346.htm

15. Brasil. Portaria Interministerial n ${ }^{\circ} 1010$, de 08 de maio de 2006. Institui as diretrizes para a Promoção da Alimentação Saudável nas Escolas de educação infantil, fundamental e nível médio das redes públicas e privadas, em âmbito nacional [Internet]. Brasília: Ministério da Saúde; Ministério da Educação, 2006.. Disponível em: http://dtr2001.saude.gov.br/sas/PORTARIAS/Port2006/GM/ GM-1010.htm

16. Brasil. Lei no 11947, de 16 de junho de 2009. Dispõe sobre o atendimento da alimentação escolar e do Programa Dinheiro Direto na Escola aos alunos da educação básica; altera as Leis nos 10.880, de 9 de junho de 2004, 11.273, de 6 de fevereiro de 2006, 11.507, de 20 de julho de 2007; revoga dispositivos da Medida Provisória no 2.178-36, de 24 de agosto de 2001, e a Lei no 8.913, de 12 de julho de 1994; e dá outras providências [Internet]. Diário Oficial da União 17 jun. 2009. Disponível em: http://www.planalto.gov.br/ccivil_03/_ato2007-2010/2009/lei/111947.htm

17. Paraná. Secretaria Estadual de Educação. Agricultura familiar - 2014 [Internet]. [acesso em 15 set. 2014]. Disponível em: http://www.comunidade.diaadia.pr.gov.br/modules/conteudo/conteudo. php?conteudo $=96$

18. Paraná. Secretaria de Estado da Educação. Diretoria de Infra Estrutura e Logística. Alimentação escolar no Paraná. Relatório organizado para apresentação no Conselho Estadual de Segurança Alimentar e Nutricional do Paraná. Curitiba: Secretaria de Estado da Educação; 2013.

19. Brasil. Ministério da Educação. Fundo Nacional de Desenvolvimento Escolar. Centro Colaborador de Alimentação escolar de Santa Catarina. Manual de orientação sobre a alimentação escolar para portadores de diabetes, hipertensão, doença celíaca, fenilcetonúria e intolerância a lactose. 2. ed. Brasília: PNAE; CECANE-SC; 2012.

20. Fortes PAC. A bioética em um mundo em transformação. Revista Bioética 2011; 19(2):319-327. 
21. Carvalho LRT, Rocha DG. Programa de aquisição de alimentos: a lente bioética na segurança alimentar. Revista Bioética 2013; 21(2):278-290.

22. Organização das Nações Unidas para a Educação, Ciências e Cultura. Declaração universal sobre bioética e direitos humanos. Brasília: Universidade de Brasília; Unesco de Bioética; 2005.

23. Neves, MCP. Bioética, biopolítica e a sociedade contemporânea. In: Porto D, Schlemper Jr B, Martins GZ, Cunha T, Hellmann F, organizadores. Bioética: saúde, pesquisa, educação. Brasília: CFM/SBB; 2014. 2 v. p. 143-67.

24. Unesco. Organização das Nações Unidas para a Educação, Ciências e Cultura. Declaração universal sobre bioética e direitos humanos. Brasília: Universidade de Brasília; Cátedra Unesco de Bioética; 2005.

25. Machado AA. Ensino jurídico e mudança social. 2. ed. São Paulo: Atlas; 2009.

26. Rubio DS. Fazendo e desfazendo direitos humanos. Santa Cruz do Sul, SC: Edunisc; 2010.

27. Valente FLS, Beghin N. Realização do direito humano à alimentação adequada e a experiência brasileira: subsídios para a replicabilidade. Brasília: ABRANDH; 2006.

28. Valente FLS, Franceschini T, Burity V. A Exigibilidade do direito humano à alimentação adequada. Brasília: ABRANDH; FAO; 2007.

29. Albuquerque MFM. A segurança alimentar e nutricional e o uso da abordagem de direitos humanos no desenho das políticas públicas para combater a fome e a pobreza. Rev. Nutr. 2009; 22(6):895-903.

30. Siqueira RL, Cotta RMM, Ribeiro RCL, Sperandio N, Priore SE. Análise da incorporação da perspectiva do direito humano à alimentação adequada no desenho institucional do programa nacional de alimentação escolar. Ciênc. Saúde Coletiva 2014; 19(1):301-310.

31. Paraná. Superintendência de Desenvolvimento Educacional. Instrução Normativa no 004/2013 SUDE/DILOG/CANE, estabelece critérios e procedimentos para o monitoramento nutricional de escolares da Rede Estadual de Ensino [Internet]. Disponível em: http://www.educacao.pr.gov.br/ arquivos/File/instrucoes/instrucaonormativa0042013dilog.pdf.

32. Hossne WS. Dos referenciais da bioética: a vulnerabilidade. Rev. Bioethikos 2009; 3(1):41-51.

33. Hossne, WS. Dos referenciais da bioética: a equidade. Rev. Bioethikos 2009; 3(2):211-216.

34. Rawls J. Uma teoria da justiça. São Paulo: Martins Fontes; 1997.

Recebido: 07/4/2014

Revisado: $14 / 7 / 2014$

Aprovado: 09/8/2014 
